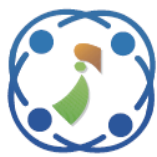

\title{
Joint Time Reversal Precoding and Spatial Diversity Technique for Acoustic Communication in Shallow Water Environment
}

\author{
Yuning Widiarti ${ }^{1,2 *} \quad$ Wirawan Wirawan $^{1} \quad$ Suwadi Suwadi $^{1}$ \\ ${ }^{1}$ Department of Electrical Engineering, Faculty of Electrical Technology, \\ Institut Teknologi Sepuluh Nopember, Surabaya, Indonesia \\ ${ }^{2}$ Shipbuilding Institute of Polytechnic Surabaya, Surabaya, Indonesia \\ * Corresponding author's Email: yuning.widiarti@ppns.ac.id
}

\begin{abstract}
Intersymbol interference (ISI) is one of the main issues in underwater acoustic communication (UAC) link development. Meanwhile, Time Reversal (TR) precoding is a promising method to mitigate ISI by means of its inherent property of spatial-temporal focusing. For the ISI problem solution and a reliable UAC link achievement, the authors propose the combination of TR precoding and maximum ratio combining (TR-MRC) techniques applied to a single-input-multi-output-OFDM (SIMO-OFDM) scheme. The system performance is observed based on Monte Carlo simulations and the analytical expressions in terms of bit-error-rate (BER) are presented to support the simulation results. There are three schemes compared, i.e.: proposed method (TR-MRC), TR-equally gain combining (TR-EGC) and simple TR. The three schemes are compared in three conditions of water depth. The analytical performance and simulation results show that the proposed method has the best performance between the other two schemes in three water depths of the towing tank. By using 2 hydrophones as a receiver array, the proposed method is proven to be superior to the simple TR. The superior performance produced up to $6 \mathrm{~dB}$ compared to the TR-EGC scheme and $8 \mathrm{~dB}$ over simple TR when the BER value is 0.1 .
\end{abstract}

Keywords: TR precoding, TR-MRC, TR-EGC, SIMO-OFDM, Shallow water environment, BER.

\section{Introduction}

Exploration of wireless communication for UAC is very challenging due to various difficulties such as attenuation, multipath propagation, limited bandwidth, rapid time variation, and severe fading. UAC utilizing acoustic waves has proven to be more resilient in conductive media such as water when compared to electromagnetic waves. The relative low sound propagation speed around $1500 \mathrm{~m} / \mathrm{sec}$ is a factor causing a long propagation delay on the receiver resulting in intersymbol interference (ISI) and these effects can reduce the system performance.

In the past decade, time-reversal (TR) communication has captured the attention in UAC. This technique is based on pivotal phenomena in physics where the principle of reciprocity and multipath effect are used to concentrate the signal energy at a certain point in space (spatial focusing) and channel impulse response (CIR) is compressed in the time domain (temporal focusing). This spatial and temporal focusing effect is useful for dealing with ISI, inter-carrier interference (ICI) and multiuser interference (MUI) [1]. Research on TR for UAC has also demonstrated [2 - 4]. While in [5] a combination of passive TR and time diversity is proposed. The time diversity of the channel is used to compensate for the channel variations.

Recently, TR has also considered a simple precoding technique that does not require complex signal processing, especially on the receiver side. Therefore, combining TR and MIMO-OFDM techniques is very advantageous for designing spectral and energy communication systems. In his research, Lo [6] proved that the maximum ratio transmission (MRT) is a precoding scheme that fully exploits the spatial diversity obtained from the transmitting antenna. This scheme maximizes SNR 
by selecting unequal weighting factors in each transmit antenna and normalizing by norm the channel coefficient vector. The TR precoding scheme [7, 8] and equal gain transmission (EGT) [9] are considered as strong candidates for the MISO system with simple pre-processing on the transmitted signal. This technique is considered to reduce complexity when compared to MRT and can be used simply in a geographically distributed antenna system. In 2005, Oestges [10] have shown that focusing on TR gain was obtained significantly in rich scattering environments. Meanwhile, in [11] it has been proven that TR with MIMO can overcome ISI and increase the channel capacity. The TR technique also provides the advantages in terms of saving power in transmitting signals by harvesting all energy from the surrounding environment and showing a large multipath diversity gain [12]. TR is also used as a simple and efficient prefiltering technique for pulse amplitude modulation (PAM) [11, 13] since it is realized over large frequency bandwidth. The TR-based precoding system is low complexity and an efficient scheme when combined with OFDM in the MISO scheme [14]. OFDM is commonly applied to deal with time dispersive channels. The combination of OFDM with TR has also been studied in $[15,16]$ and is proven to be a simple and efficient method for improving the performance of the UAC system.

To our knowledge, no existing research addresses the use of TR precoding to SIMO-OFDM in the underwater acoustic channel. In this paper, we propose the application of TR-MRC in SIMOscheme and present the performance analysis of TRMRC and compare it with other precoding schemes. The conclusions obtained are further confirmed through computer simulations. There are several contributions offered in this paper as follows:

1. Joint TR precoding and spatial diversity techniques in the SIMO-OFDM scheme.

2. Investigation of the performance of TR precoding and spatial diversity techniques in shallow water environment based on real measurement of the CIR.

3. Analysis of TR-MRC precoding and other precoding schemes performance in terms of BER. The rest of this paper is organized as follows: In section 2, the related works are presented. In section 3 , a system model of joint TR precoding and diversity combining techniques in the SIMO-OFDM scheme is introduced. Monte Carlo simulation results are also presented to confirm the theoretical results in section 4. In this section, the average received signal-to-noise ratio (SNR) and the average
BER of every system are computed and analyzed. Finally, the conclusions are drawn in section 5.

\section{Related works}

Several studies have proven that the precoding technique is very suitable to be applied to wireless communication systems such as the TR and MRT precoding scheme. In 1999, Lo proposed a classical precoding scheme [6]. In the scheme, Lo moves the traditional matched filter from the receiver side to the transmitter side. This scheme maximizes the SNR by selecting non-uniform weighting factors in each branch of the antenna and normalizing them with the norm vector channel coefficient. This implies that in the MISO-OFDM system the normalization factor for each subcarrier and each antenna increases the computational complexity. Later, this precoding scheme is proven to be equivalent to the optimal SVD precoding scheme under some normalization assumptions.

In [9], the authors introduce the EGT technique which allows transmission with a unitary gain on every link. In his research, precoding only consisted of phase rotation to avoid any power imbalance between the signals transmitted by each antenna. By considering several antennas at the receiver, combining technique can be performed with a precoding technique to take the advantage of the diversity of signals received by the array antenna. However, in this study, it is assumed that signal propagation over a single-band flat fading channel without taking into account the frequency diversity. By considering more wideband transmission systems, the exploitation of diversity and performance can vary from one precoding scheme to another.

This paper is motivated by research in $[14,15]$. In [14], the authors apply TR precoding in the MISO-OFDM system and use several transmit antennas on the transmitter side. By utilizing the TR focusing property, the equivalent TR-OFDM transmission channel can be shorter than the conventional OFDM channel. This research also proves that TR can reduce the guard interval of OFDM symbols and also increases the system spectral efficiency for a large number of transmit antenna. TR performance will increase with the increasing number of transmit antennas. The system performance of TR is superior compared to the MRT scheme because of their ability to reduce the guard interval length. However, this paper does not include the detailed theoretical expressions of BER for the number of transmit antennas. 
Similarly to [14], the authors in [15] are motivated to apply the TR precoding in MISOOFDM. The authors compare the performance of simple TR precoding and EGT schemes by using simulation and generic theoretical expressions of the average BER and the system capacity for a number of transmit antennas. Simulation results show that the TR scheme is superior to EGT with a relatively large number of transmit antennas.

In contrast to performed studies in [14, 15], this paper proposes the joint TR precoding and spatial diversity combining technique (TR-MRC) in the SIMO-OFDM scheme. The purposes of this study are to reduce the effect of ISI arising from multipath fading and also improve the performance of the UAC system. This paper also shows the real measurement procedure in a towing tank. The impulse responses derived from the real measurement with 3 depth variations. In this paper, the TR-MRC is considered as a benchmark and other diversity techniques such as simple TR and TR-equally gain combining (TR-EGC) are presented as comparison techniques. The authors also present the theoretical expressions in terms of BER for different schemes. In this paper, MRT and EGT are used to mention the terms of spatial diversity techniques in the MISO scheme. Meanwhile, the terms change to MRC and EGC respectively in the SIMO scheme.

\section{Model system}

In this section, the TR precoding principle is described as well as its application in UAC.

\subsection{TR Precoding application in UAC}

As discussed in the previous section, UAC is constrained by the data rates due to the ISI effects which result in decreased performance and system capacity. The system capacity can be increased by using the SIMO structure [18]. In wireless communication application, TR consists of prefiltering the transmitted signal by the time-reversed and conjugated version of the CIR between transmitter and receiver antenna. Therefore, TR precoding requires knowledge of the channel on the transmitter side as well as the channel feedback. The time focusing property of TR allows the realization of communication over an equivalent channel with lower ISI than that produced by the propagation channel. This is what distinguishes between the conventional spatial diversity techniques such as MRC and EGC [21] with the proposed method in this paper. The more receiving antennas used, the greater focusing in the time and spatial domains obtained. In UAC, the antennas of the receiver are replaced by a hydrophone array as the sound signal sensors.

Let the CIR of UAC between the transmitter (sound source) and the i-th hydrophone is denoted as $h_{i}(t)$ and $s(t)$ is a notation for transmitted data symbol. While $n(t)$ represents a white Gaussian noise. In TR precoding, the transmitted signal is filtered by $h_{i}{ }^{*}(-t)$ firstly, and thus, the received signal can be expressed as:

$$
r(t)=h_{i}(t) * h_{i}^{*}(-t) * g(t) s(t)+n(t)
$$

The notation $*$ means the convolution product and $g(t)$ shows the shape filter. The equivalent channel of TR communication can be derived from the autocorrelation of the CIR, i.e. $h_{i}(t)^{*} h_{i}{ }^{*}(-t)$.

\subsubsection{Measurement description}

The impulse response is a characterization of underwater acoustic channels which is useful for predicting and evaluating the performance and transmission bandwidth of communication systems for certain underwater acoustic channel conditions [20]. In this research, the impulse response is conducted in a towing tank belonging to the Indonesian Hydrodynamic Laboratory (IHL) with a dimension of $12 \mathrm{~m} \mathrm{x} 200 \mathrm{~m}$ and a depth of $6 \mathrm{~m}$. An underwater speaker has been placed at a depth of 1.5 $\mathrm{m}$, and this depth varies for system performance evaluation. The speaker is $20 \mathrm{~m}$ away from the back wall. As a receiver, the hydrophone array is placed $70 \mathrm{~m}$ apart from the source. At the time of measurement, the medium condition is uniform, in which there are no waves and no sources of transient noise. The experimental setup is presented as shown in Fig. 1.

Probe signal is generated by using the PC software equipped with a sound card and power amplifier. The conversion of an electrical signal into an acoustic signal is the task of the underwater speaker. The underwater speaker used is Aquasonic AQ339 with the following specifications: $135 \mathrm{~W}$ of power, a $4 \Omega$ impedance, and a frequency of $20 \mathrm{~Hz}-$ $17 \mathrm{kHz}$. Meanwhile, the received signal is captured by hydrophones that have the following specifications: frequency ranges of $1 \mathrm{~Hz} \sim 100 \mathrm{kHz}$, a sensitivity of $-190 \mathrm{dBre}: 1 \mathrm{~V} / \mu \mathrm{Pa}(+4 \mathrm{~dB}, 20 \mathrm{~Hz}-$ $4 \mathrm{kHz}$ ), and an internal capacitance of $25 \mathrm{nF}$. The distance between the hydrophones in data retrieval is influenced by the recorded wavelength and the distance of the source to the hydrophone array. The 


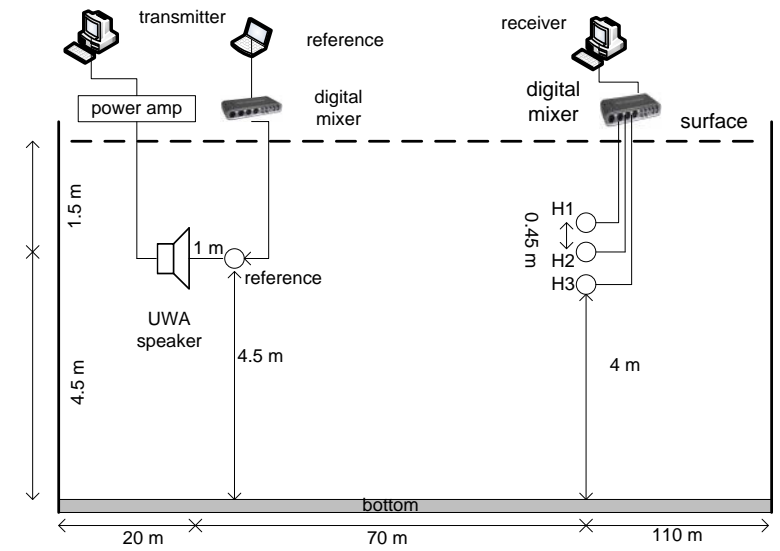

Figure. 1 Experimental setup for CIR measurement

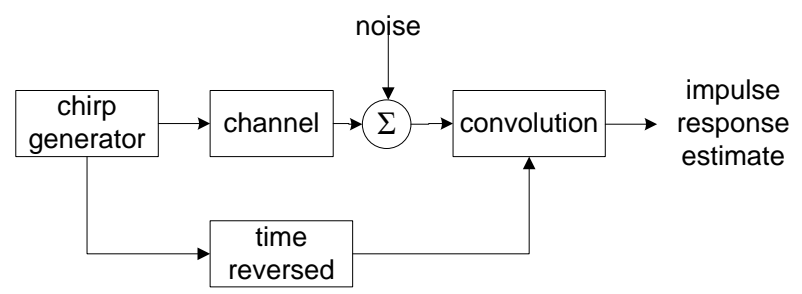

Figure. 2 CIR estimation

algorithm is denoted as $d<\lambda_{\min } / 2$ with $d$ being the distance between hydrophones, while $\lambda_{\min }$ is the minimum wavelength of the captured signal.

By considering the highest carrier frequency of the chirp signal, the distance between the hydrophones must be less than $0.5 \mathrm{~m}$. In this measurement, the distance between hydrophones is set at $0.45 \mathrm{~m}$, to make it safe from aliasing.

\subsubsection{CIR measurement}

The impulse response is obtained by sending the probe signal firstly. The probe signal used is a linear frequency modulation signal (chirp). Chirp signal to be transmitted has a duration of $100 \mathrm{msec}$ and frequency sweep from $1000 \mathrm{~Hz}$ to $15000 \mathrm{~Hz}$. The chirp signal used has the signal equation as follows:

$$
x(t)=A \cos (\omega t+\varphi)
$$

$A$ stand for the amplitude of the signal, $\omega$ is the angular frequency and $\varphi$ is an initial phase.

The transmitted chirp is captured by the hydrophone array. To get the estimated CIR, the procedure can be performed as illustrated in Fig. 2. This estimated CIR is then used to simulate the proposed method. There are 3 hydrophones as a receiver array, so there are 3 branches in the receiver side.

\subsection{TR precoding for the SIMO-OFDM system}

Referring to [19] that TR OFDM communication can be expressed in the time-domain or frequencydomain. Therefore, the time-domain filters can be replaced with the frequency-domain filter without changing the performance and the received symbol expression. In this study, UAC is considered as quasi-static, which is constant on several OFDM symbols.

If the precoding technique is applied to each subcarrier, then the received symbol can be expressed as follows :

$$
R_{p, q}=\sum_{l=1}^{N_{r}} H_{p, l} V_{p, l} S_{p, q}+N_{p, q}
$$

By using the assumption of perfect channel estimation and synchronization system for UAC used, the received symbol in Eq. (3) with the $p$-th subcarrier and $q$-th OFDM symbol is expressed in the frequency domain. In the equation $H_{p, l}$ denotes the complex channel coefficient. In this term $h_{p}$ is a channel vector of size $N_{r}$ (number of hydrophones).

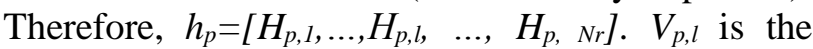
precoding factor that depends on the hydrophone index $l$ as well as on the channel response, $S_{p, q}$ is the data symbol and $N_{p, q}$ is the noise term associated with the $p$-th subcarrier of the $q$-th OFDM symbol. The noise variance is the same overall sub-carriers and notated as $\sigma_{q}^{2}$.

TR precoding also allows the realization of a simple SIMO-OFDM system. As shown in Fig.3, only the knowledge of the channel between transmitter and receiver is required. By applying the appropriate precoding to the transmitted signal, then the signals are received and added coherently in the receiver. As seen in Fig. 4, the precoding and CIR vector related to data symbol $S_{p, q}$ are $V_{p, l}$ and $H_{p, l}$ respectively.

\subsubsection{Joint TR Precoding and MRC}

In the TR-MRC system, the signal transmitted is firstly pre-filtered by the time-reversed and conjugated version of impulse responses. Then, the resulting signals are added coherently in the receiver. In the SIMO-OFDM system, the optimal MRC precoding is applied to the transmitted data symbols $S_{p, q}$. Thus, TR-MRC precoding can be written as follows:

$$
V_{p, l}=\frac{H_{p, l}^{*}}{\sqrt{\lambda_{p}}}
$$




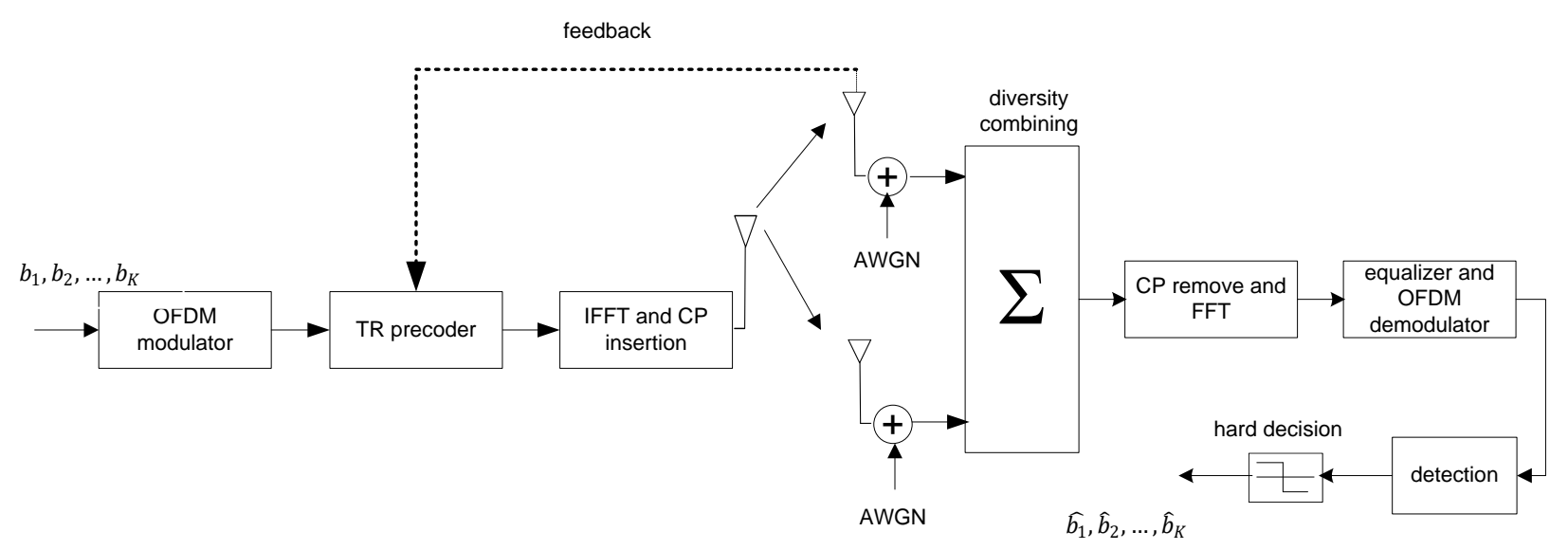

Figure. 3 SIMO-OFDM transmission with TR precoder

where $\sqrt{\lambda_{p}}=\sqrt{\sum_{l=1}^{N_{r}}\left|H_{p, l}\right|^{2}}$ is the singular value of $h_{p}$. The factor $\sqrt{\lambda_{p}}$ is chosen to get the optimum received SNR. When $\mathrm{H}_{p, l}$ follows an independent normalized distribution, then $E\left[\lambda_{p}\right]=N_{r}$. By substituting Eq. (4) in Eq. (3), then the received symbol on each subcarrier can be expressed as follows:

$$
\begin{aligned}
R_{p, q} & =\frac{\sum_{l=1}^{N_{r}}\left|H_{p, l}\right|^{2}}{\sqrt{\lambda_{p}}} S_{p, q}+N_{p, q} \\
& =\sqrt{\sum_{l=1}^{N_{r}}\left|H_{p, l}\right|^{2}} S_{p, q}+N_{p, q}
\end{aligned}
$$

In Eq. (5) the equivalent channel coefficient $\left|H_{p, l}\right|^{2}$ is real which indicates that the received symbols do not undergo any phase rotation. Therefore, in this SIMOOFDM scheme, the instantaneous received SNR obtained over the subcarrier due to the TR-MRC precoding can be written as follows:

$$
\gamma_{\mathrm{TR}-\mathrm{MRC}}=\left(\sum_{\mathrm{l}=1}^{\mathrm{N}_{\mathrm{r}}}\left|\mathrm{H}_{\mathrm{p}, \mathrm{l}}\right|^{2}\right) \bar{\gamma}
$$

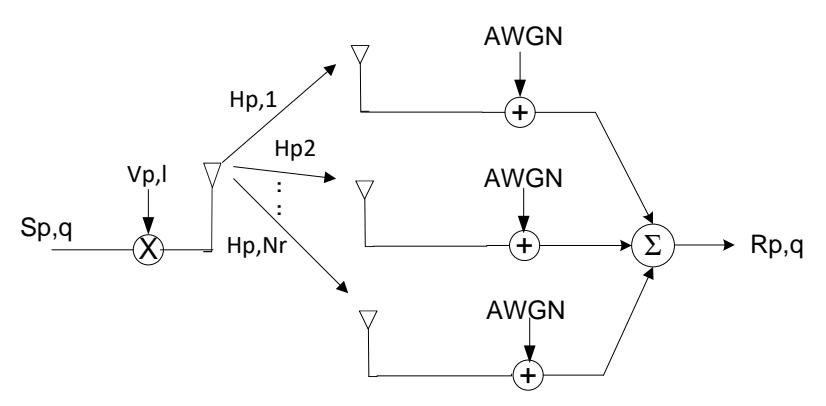

Figure. 4 Precoding of modulated symbol over the $p$ th subcarrier
$\bar{\gamma}=\frac{P_{t}}{\sigma^{2}}$ is the average transmit SNR of each branch.

\subsubsection{Joint TR precoding and EGC}

EGC technique resembles MRC. The difference is that there is no weighting in the EGC scheme, and there is a possibility to put together high-quality signals (interference and noise-free) and low-quality signals. EGC scheme also aims to maximize the received SNR [9]. In this scheme, the phase modification is performed on complex symbols before they are transmitted. In SIMO-OFDM, the precoding vector for TR-EGC can be written as follows:

$$
\begin{aligned}
V_{p, l}= & \frac{e^{j \theta H_{p, l}^{*}}}{\sqrt{N_{r}}} \\
& =\frac{\sqrt{e^{-j \theta H_{p, l}}}}{\sqrt{N_{r}}}
\end{aligned}
$$

$\theta H_{p, l}^{*}$ and $\theta H_{p, l}$ are the argument of channel fading coefficient $H_{p, l}^{*}$ and $H_{p, l}$ respectively. Different from the MRC case, the instantaneous power associated with each subcarrier does not vary with the precoding factor because it only consists of phase rotation. When Eq. (7) is substituted into Eq. (1), then the received symbol on each subcarrier can be expressed as follows:

$$
\begin{aligned}
R_{p, q} & =\sum_{l=1}^{N_{r}} H_{p, l} \frac{e^{-j \theta H_{p, l}}}{\sqrt{N_{r}}} S_{p, q}+N_{p, q} \\
& =\frac{1}{N_{r}} \sum_{l=1}^{N_{r}}\left|H_{p, l}\right| S_{p, q}+N_{p, q}
\end{aligned}
$$


Similar to TR-MRC scheme in SIMO-OFDM, the received signal is also multiplied by the real factor $\sum_{l}^{N_{r}}\left|H_{p, l}\right|$, and therefore, the transmitted signal can be simply demodulated and the received SNR along the $p$-th subcarrier can be expressed as follows:

$$
\gamma_{\mathrm{TR}-\mathrm{EGC}}=\left(\sum_{\mathrm{l}=1}^{\mathrm{N}_{\mathrm{r}}}\left|\mathrm{H}_{\mathrm{p}, \mathrm{l}}\right|\right)^{2} \frac{\bar{\gamma}}{\mathrm{N}_{\mathrm{r}}}
$$

\subsubsection{TR precoding}

In wireless communication applications, TR involves the process of pre-filtering a signal with the time-reversed and conjugated version of CIR between the transmitter and receiver. Referring to Eq. (1), equivalent channels are obtained from the convolution between discrete complex-baseband CIR and time-reversed and conjugated versions of CIR. As a consequence, the equivalent channel is made of a central peak of high amplitude and some side lobes. The ability of time focusing on TR can be identified from the peak of the autocorrelation channel which is getting sharper and narrower and the side lobes are decreasing. Therefore, TR can compress the time dispersion and ISI between symbols can be minimized. To overcome the residual ISI with limited bandwidth and a limited number of receiving elements, a multicarrier system is considered efficient to implement.

In this precoding scheme, the complex modulated symbol $S_{p, q}$ on each subcarrier is multiplied by the conjugate of the CIR coefficient $H_{p, l}{ }^{*}$. In accordance, the precoding factor can be written as follows :

$$
V_{p, l}=\frac{H_{p, l}^{*}}{\sqrt{N_{r}}}
$$

Then, the transmitted symbol $S_{p, q}$ over the $l$-th hydrophone and $p$-th subcarrier is:

$$
\begin{aligned}
R_{p, q} & =\sum_{l=1}^{N_{r}} H_{p, l} \frac{H_{p, l}^{*}}{\sqrt{N_{r}}} S_{p, q}+N_{p, q} \\
& =\frac{1}{\sqrt{N_{r}}} \sum_{l=1}^{N_{r}}\left|H_{p, l}\right|^{2} S_{p, q}+N_{p, q}
\end{aligned}
$$

The instantaneous receiving signal-to-noise ratio (SNR) over the subcarrier $p$ due to TR precoding can be written as:

$$
\gamma_{T R}=\left(\sum_{l=1}^{N_{r}}\left|H_{p, l}\right|^{2}\right)^{2} \frac{\bar{\gamma}}{N_{r}}
$$

Eq. (12) shows that increasing the number of receiver elements will raise the SNR received because the CIR coefficients are added coherently on the receiver side.

\subsection{BER of system}

In this section, the calculation of the average bit error rate (BER) is performed to evaluate the system performance. In the previous sub-section, it has been shown that the signal received on each subcarrier depends on the precoding factor used. In addition, the precoding function makes signal entry dependent on channel realization and affects the level of power received.

For BER calculations, the parameters in Eqs. (6), (9), and (12) are used. With the same transmit SNR value $(\bar{\gamma})$, the different received SNR values will be obtained based on the precoding function used. The performance evaluation on each SIMO scheme in terms of the average BER calculation for QPSK modulation is a function of transmitting SNR $(\bar{\gamma})$. If the received SNR is equal to $x \bar{\gamma}$, then the average BER can be calculated by this following equation:

$$
\operatorname{BER}(\bar{\gamma})=\frac{1}{2} \int_{-\infty}^{\infty} \mathrm{p}(\mathrm{x}) \cdot \operatorname{erfc}(\sqrt{\mathrm{x} \bar{\gamma}})
$$

where erfc stand for complementary error function, and $x$ is a random variable with a probability density function (pdf) denoted by $p(x)$ which depends on the channel and precoding factor used.

\subsubsection{BER of TR-MRC}

The received SNR for TR-MRC scheme is denoted as $\gamma_{T R-M R C}$, therefore, it can be rewritten as follows:

$$
\begin{aligned}
\gamma_{T R-M R C} & =\left(\sqrt{\sum_{l=1}^{N_{r}}\left|H_{p, l}\right|^{2}}\right)^{2} \bar{\gamma} \\
& =\sum_{l=1}^{N_{r}}\left|H_{p, l}\right|^{2} \bar{\gamma}=x \bar{\gamma}
\end{aligned}
$$

With $x=\sum_{l=1}^{N_{r}}\left|H_{p, l}\right|^{2}$, then the average BER can be calculated by Eq. (14). Assuming $x$ is a sum of independent identically distributed Gaussian variables with standard deviation $(\sigma)=\frac{1}{\sqrt{2}}$. Therefore, $x$ follows the Gamma distribution with a 
shape parameter of $\mathrm{N}_{\mathrm{r}}$ and a scale parameter of $2 \sigma^{2}=1$. The pdf of $x$ can be expressed as follows:

$$
p(x)=\left\{\begin{array}{cc}
\frac{1}{\Gamma\left(N_{r}\right)} x^{N_{r}-1} e^{-x} & \text { for } x>0 \\
0 & \text { else }
\end{array}\right.
$$

Where $\Gamma$ (.) stands for the Gamma function. If Eq. (15) is substituted into Eq. (13), then the following equation will be obtained :

$B E R_{T R-M R C}=\int_{0}^{+\infty} \frac{1}{\Gamma\left(N_{r}\right)} x^{N_{r}-1} e^{-x} \frac{1}{2} \operatorname{erfc}(\sqrt{x \bar{\gamma}}) d x$

By using the Taylor series $\mathrm{e}^{-\mathrm{x}}$, the formula becomes:

$$
\begin{array}{r}
B E R_{T R-M R C}(\bar{\gamma})=\frac{1}{2 \Gamma\left(N_{r}\right)} \sum_{l=0}^{+\infty} \frac{(-1)^{l}}{l !} \\
\int_{0}^{+\infty} x^{l+N_{r}-1} \operatorname{erfc}(\sqrt{x \bar{\gamma}}) d x
\end{array}
$$

And by replacing the variable as $\mathrm{k}=\sqrt{\mathrm{x} \bar{\gamma}}$ and using the formula:

$$
\int_{0}^{+\infty} k^{2 y-1} \operatorname{erfc}(k) d k=\frac{\Gamma\left(y+\frac{1}{2}\right)}{2 \sqrt{\pi} y}
$$

With $y=l+N_{r}$, then the Eq. (17) becomes:

$$
\begin{aligned}
B E R_{T R-M R C}(\bar{\gamma})= & \frac{1}{\Gamma\left(N_{r}\right)} \sum_{l=0}^{+\infty} \frac{(-1)^{l}}{l !}\left(\frac{1}{\bar{\gamma}}\right)^{l+N_{r}} \\
& \frac{\Gamma\left(l+N_{r}+\frac{1}{2}\right)}{2 \sqrt{\pi}\left(l+N_{r}\right)}
\end{aligned}
$$

From Eq. (19) it can be deduced that the asymptotic average BER is obtained for high SNR. The average BER is expressed as a sum of the power $\bar{\gamma}$. The limit for high SNR is related to the highest power value. Therefore, the asymptotic BER is obtained when $l=$ 0 .

$$
\lim _{\bar{\gamma} \rightarrow+\infty} B E R_{T R-M R C}(\bar{\gamma})=\frac{1}{\Gamma\left(N_{r}\right)} \frac{\Gamma\left(N_{r}+\frac{1}{2}\right)}{2 N_{r} \sqrt{\pi}}\left(\frac{1}{\bar{\gamma}}\right)^{N_{r}}
$$

$$
\begin{gathered}
=\frac{1}{\left(N_{r}-1\right) !} \frac{\left(2 N_{r}\right) ! \sqrt{\pi}}{2^{2 N_{r}}\left(N_{r}\right) !} \frac{1}{2 N_{r} \sqrt{\pi}}\left(\frac{1}{\bar{\gamma}}\right)^{N_{r}} \\
=\frac{\left(2 N_{r}\right) !}{2^{2 N_{r}+1}\left(N_{r} !\right)^{2}}\left(\frac{1}{\bar{\gamma}}\right)^{N_{r}}
\end{gathered}
$$

\subsubsection{BER of TR-EGC}

In the TR-EGC, the received SNR which is expressed in Eq. (9) can be rewritten as follows:

$$
\gamma_{T R-E G C}=\left(\sum_{l=1}^{N_{r}}\left|H_{p, l}\right|\right)^{2} \frac{\bar{\gamma}}{N_{r}}=\frac{z^{2} \bar{\gamma}}{N_{r}}
$$

with $z=\sum_{l=1}^{N_{r}}\left|H_{p, l}\right|$, and therefore, the average BER can be calculated by the following equation:

$$
B E R_{T R-E G C}(\bar{\gamma})=\frac{1}{2} \int_{-\infty}^{+\infty} p(z) \operatorname{erfc}\left(z \sqrt{\frac{\bar{\gamma}}{N_{r}}}\right)
$$

The obtained pdf is the sum of Rayleigh distributed random variables. This paper uses 3 hydrophones $\left(N_{r}\right.$ = 3). Modulus $\left|H_{p, l}\right|$ follows the Rayleigh distribution and it is known that such a distribution will be equal to zero for $t<0$, so the pdf can be written as follows:

$$
p(z)=\frac{e^{-\frac{z^{2}}{4 \sigma^{2}}}}{\sigma^{3}}\left\{\sqrt{\pi} \operatorname{erf}\left(\frac{z}{2 \sigma}\right)\left(\frac{z^{2}}{4}-\frac{\sigma^{2}}{2}\right)+\frac{z \sigma}{2} e^{-\frac{z^{2}}{4 \sigma^{2}}}\right\}
$$

where erf stand for the error function. By substituting Eq. (23) in Eq. (22), the average BER formula is obtained as follows:

$$
\begin{aligned}
& B E R_{T R-E G C}(\bar{\gamma})= \\
& \frac{1}{4 \sigma^{3}} \sum_{l=0}^{+\infty} \sum_{n=0}^{+\infty} \frac{(-1)^{l+n}}{l ! n !(2 n+1) 2^{2 l+2 n+1} \sigma^{2 l+2 n+1}} \\
& \left\{\left(\frac{2}{\bar{\gamma}}\right)^{l+n+2} \frac{\Gamma\left(l+n+\frac{5}{2}\right)}{2 \sqrt{\pi}(l+n+2)}-2 \sigma^{2}\left(\frac{2}{\bar{\gamma}}\right)^{l+n+1} \frac{\Gamma\left(l+n+\frac{3}{2}\right)}{2 \sqrt{\pi}(l+n+1)}\right\}
\end{aligned}
$$

For high SNR, BER TR-EGC $_{\text {can be expressed as }}$ follows:

$$
\lim _{\bar{\gamma} \rightarrow+\infty} B E R_{T R-E G C}(\bar{\gamma})=\frac{1}{16 \sigma^{4}} \frac{1}{\bar{\gamma}^{2}}=\frac{1}{4} \frac{1}{\bar{\gamma}^{2}}
$$

\subsubsection{BER of TR}

The received SNR in the SIMO-OFDM scheme which refers to Eq. (11) can be rewritten as in the following equation: 
Table 1. OFDM specification

\begin{tabular}{|c|c|}
\hline Parameter & Value \\
\hline Sampling rate (fs) & $48 \mathrm{kHz}$ \\
\hline Number of FFT (NFFT) & 64 \\
\hline Number of used subcarriers & 52 \\
\hline Number of a cyclic prefix (Ncp) & 16 \\
\hline $\begin{array}{c}\text { Number of the transmitted } \\
\text { symbol }\end{array}$ & 1000 \\
\hline $\begin{array}{c}\text { The maximum frequency of chirp } \\
\left(\mathrm{f}_{1}\right)\end{array}$ & $1 \mathrm{kHz}$ \\
\hline Minimum frequency of chirp $\left(\mathrm{f}_{0}\right)$ & $15 \mathrm{kHz}$ \\
\hline Modulation scheme & QPSK \\
\hline
\end{tabular}

$$
\bar{\gamma}_{T R}=\left(\sum_{l=1}^{N_{r}}\left|H_{p, l}\right|^{2}\right)^{2} \frac{\bar{\gamma}}{N_{r}}=\frac{x^{2} \bar{\gamma}}{N_{r}}
$$

By using Eq. (22) and substituting $x=\sum_{l=1}^{N_{r}}\left|H_{p, l}\right|^{2}$ into $z$, then the average BER can be obtained. Meanwhile, the pdf of $x$ is obtained from equation (15) which follows the Gamma distribution. After going through the formation in the Taylor series and changing a variable on the expression, the following equation is obtained:

$$
\begin{aligned}
B E R_{T R}(\bar{\gamma})= & \frac{1}{2 \Gamma\left(N_{r}\right)} \sum_{l=0}^{+\infty} \frac{(-1)^{l}}{l !} \\
& \frac{\Gamma\left(\frac{l+N_{r}+1}{2}\right)}{2 \sqrt{\pi}\left(\frac{l+N_{r}}{2}\right)}\left(\sqrt{\frac{N_{r}}{\bar{\gamma}}}\right)^{l+N_{r}}
\end{aligned}
$$

At high SNR, the average BER can be written as follows:

$$
\lim _{\bar{\gamma} \rightarrow+\infty} B E R_{T R}(\bar{\gamma})=\frac{1}{2^{N_{r}+1}\left(\frac{N_{r}}{2}\right) !}\left(\frac{N_{r}}{\bar{\gamma}}\right)^{\frac{N_{r}}{2}}
$$

\section{Simulation results and discussion}

The CIR measurement is performed with the distance between the transmitter and receiver $70 \mathrm{~m}$ apart is as shown in Fig. 1. Depths are varied, i.e.: $1.5 \mathrm{~m}, 2.5 \mathrm{~m}$, and $3.5 \mathrm{~m}$. To get the CIR, the chirp signal probe is transmitted from the source and captured by the receiver array. The mechanism to get the estimated impulse response as shown in Fig. 2. By using the convolution process between the original chirp signal and the time-reversed signal, the CIR obtained is illustrated in Fig. 5.

The impulse response at $70 \mathrm{~m}$ distance with a depth of $1.5 \mathrm{~m}$ has higher relative power, especially at the ch.3 (the $3^{\text {rd }}$ hydrophone), while the relative power at ch. 2 and ch.3 is smaller. At a depth of $2.5 \mathrm{~m}$, the relative power obtained on three channels is almost the same. The relative power at a depth of 3.5 $\mathrm{m}$ is smaller than the relative power at a depth of 1.5 $\mathrm{m}$ and $2.5 \mathrm{~m}$, and at this depth, each channel has a delay difference between one channel and the others. However, from the three depths observed, the CIR pattern produced was almost the same and the relative power generated did not have a significant difference. In this section, a Monte Carlo simulation is presented to evaluate the reliability of the TRMRC system and compare it with TR-EGC and conventional TR techniques. The performance parameter evaluated is BER against the transmitting SNR. OFDM specifications used for this simulation can be shown as in Table 1 .

It has been discussed in the previous section about the SNR received in each scheme and the asymptotic BER for a number of $\mathrm{N}_{\mathrm{r}}$ elements.

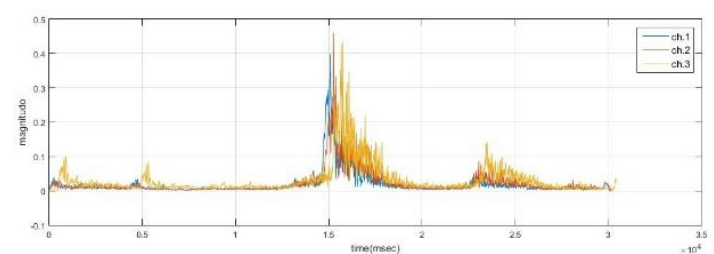

(a)

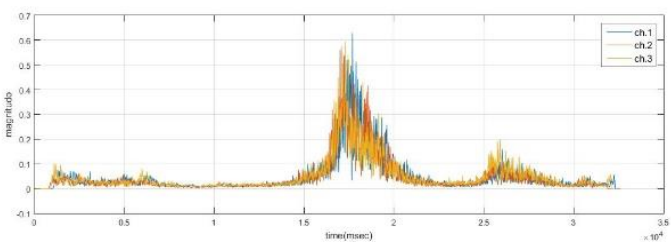

(b)

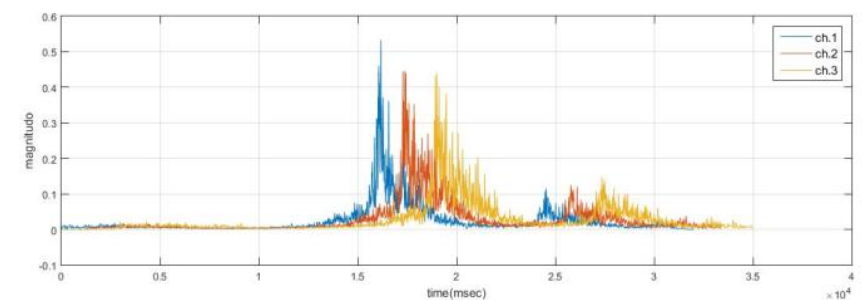

(c)

Figure. 5 CIR at $70 \mathrm{~m}$ distance: (a) depth of $1.5 \mathrm{~m}$, (b) depth of $2.5 \mathrm{~m}$, and (c) depth of $3.5 \mathrm{~m}$ 


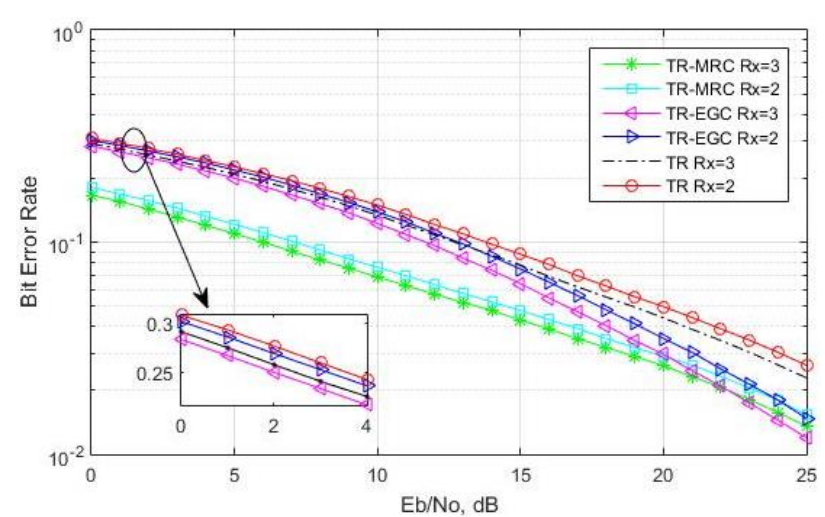

Figure. 6 BER at $70 \mathrm{~m}$ and a depth of $1.5 \mathrm{~m}$

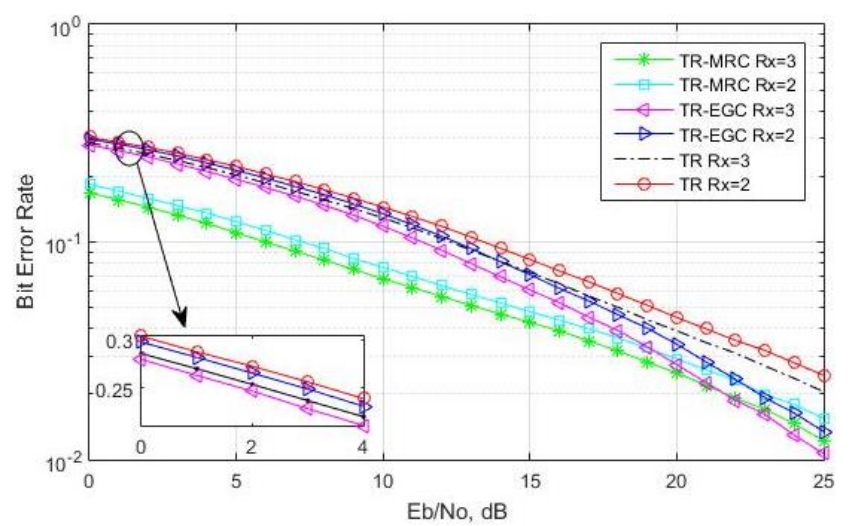

Figure. 7 BER at $70 \mathrm{~m}$ and a depth of $2.5 \mathrm{~m}$

It appears that the realization of the channel on the precoding factor is very influential, and therefore it has an effect on the received power. In conventional TR, the factor is a squared sum of the squared modulus of the channel coefficients, so it appears that some channel coefficients have a power of 4 when compared with the SNR received on TR-EGC and TR-MRC with a power equal to 2 . However, after observing the obtained BER expression, then the TR-MRC precoding has a power term of $\mathrm{N}_{\mathrm{r}}$ and TR precoding has a power term of $\mathrm{N}_{\mathrm{r}} / 2$. In TR-EGC there is no general closed-form for asymptotic BER. In general, the simulation results show that TRMRC has a superior performance compared to TREGC due to the weighting factor $\frac{1}{\bar{\gamma}^{2}}$. In the TR scheme, the asymptotic BER obtained becomes $\frac{1}{4 \bar{\gamma}}$ and thus, TR benefits from neither diversity gain nor SNR gain compared to the other two schemes.

In the Monte Carlo simulation, it is assumed that the transmitted power is the same and the noise power does not change in all precoding schemes. The resulting BER curve is versus Eb/No. Eb/No is obtained from the transmitted $\operatorname{SNR}(\bar{\gamma})$, i.e.

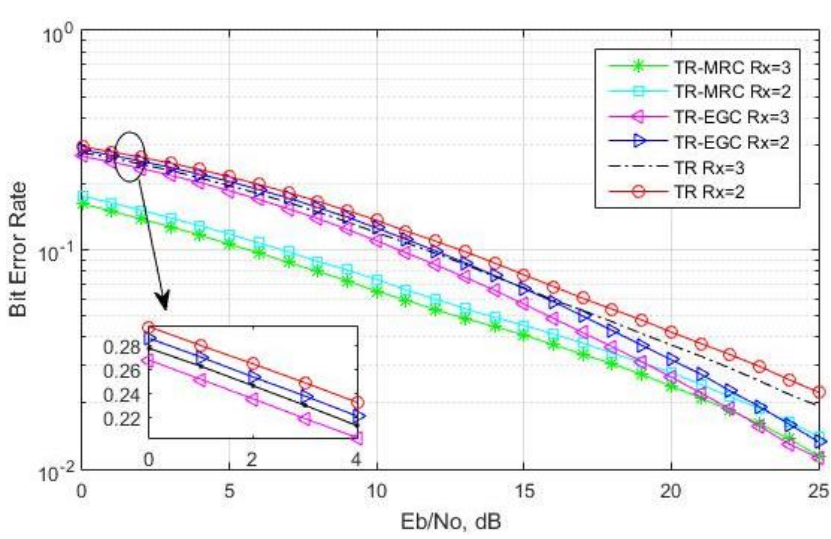

Figure. 8 BER at $70 \mathrm{~m}$ and a depth of $3.5 \mathrm{~m}$

$(\mathrm{Eb} / \mathrm{No}) \mathrm{dB}=\mathrm{dB}-3 \mathrm{~dB}$ for QPSK modulation. The cyclic prefix (cp) size used is also the same for all precoding. Fig. 6 shows BER for a depth of $1.5 \mathrm{~m}$ at a distance of $70 \mathrm{~m}$ between the source and receiver array. The BER curve of TR-MRC is superior compared to two other schemes, i.e. TR and TREGC. At 0 to $20 \mathrm{~dB}$ the performance of TR-MRC is significantly superior to TR-EGC. In general, the BER curve with $N_{r}=3$ is superior to the BER curve with $N_{r}=2$. The number of hydrophones $\left(N_{r}\right)$ needed is smaller when compared to previous studies [14] and [15]. At SNR 0-22 dB, TR-MRC is superior when compared to TR-EGC and simple TR. When the BER value is 0.1 , there is a difference of 6 $\mathrm{dB}$ between TR-MRC and TR-EGC. Meanwhile, the difference between simple TR and TR-MRC is $8 \mathrm{~dB}$. However, at $\mathrm{SNR} \geq 23 \mathrm{~dB}$, TR-EGC was slightly superior to the TR-MRC scheme. At low SNR, i.e. 0-14 dB the conventional TR looks slightly superior to the TR-EGC scheme, but when SNR is above 15 $\mathrm{dB}$ the TR-EGC performance is more dominant than simple TR.

If three depths of the towing tank are observed, there is actually no significant difference in BER results due to the CIR pattern produced. This shows that in a controlled environment such as in the towing tank, the dynamics and water conditions are relatively constant, this is shown in Figs. 7 and 8 . BER curves at depths of $1.5 \mathrm{~m}, 2.5 \mathrm{~m}$, and $3.5 \mathrm{~m}$ show that the best TR-MRC performance between the other two schemes is at SNR of less than $20 \mathrm{~dB}$. At SNR above $20 \mathrm{~dB}$, the TR-EGC BER curve coincides with TR-MRC, even when SNR above 23 dB TR-EGC looks slightly superior to the TR-MRC scheme. If observed closely, of the three depths the performance of the TR-MRC is best at a depth of $3.5 \mathrm{~m}$. 


\section{Conclusion}

This paper has investigated the application of TR precoding in the SIMO-OFDM scheme and could be adopted for the implementation of massive MIMO in underwater acoustic channels. In SIMOOFDM schemes the authors have presented the new theoretical expressions of the BER average of the simple TR, TR-MRC, and TR-EGC. The formula supports the simulation results. This shows that by increasing the number of hydrophones at the receiver, the system performance will be increased. Monte Carlo simulations prove that TR-MRC has the best performance compared to the other schemes, especially at low SNR i.e. between $0-23 \mathrm{~dB}$ in all depth conditions. TR-MRC is proven to be superior by $6 \mathrm{~dB}$ compared to TR-EGC and $8 \mathrm{~dB}$ compared to simple TR when BER reaches 0.1 . Only by using 2 hydrophones, TR-MRC has a performance that is superior to simple TR and TR-EGC in a shallow water environment in the SIMO-OFDM scheme.

\section{Acknowledgments}

The authors extend their gratitude to IHL which has provided support to perform the measurements and also to the Ministry of Research, Technology and Higher Education of the Republic of Indonesia for the Domestic Postgraduate Education Scholarships (BPPDN 2015-2019)

\section{References}

[1] Y. Han, Y. Chen, B. Wang, and K. J. R. Liu, "Time Reversal Massive Multipath Effect: A Single Antenna "Massive MIMO" Solution", IEEE Transactions on Communications, Vol. 64, No. 8, pp. 3382-3394, 2016.

[2] L. Sun, B.W. Chen, H.S. Li, T. Zhou, and R. Li, "Time Reversal Acoustic Communication Using Filtered Multitone Modulation", Sensors, Vol.15, No.9, pp.22357-23554, 2015.

[3] S. Kim, S.U. Son, H. Kim, K.H. Choi, and J.W. Choi, "Estimate of Passive Time Reversal Communication Performance in Shallow Water", Applied Sciences, Vol. 8. No.1, pp. 2331, 2017.

[4] A. Zhao, C. Zeng, J. Hui, L. Ma, and X. Bi, "An Underwater Time Reversal Communication Method Using Symbol-Based Doppler Compensation with A Single Sound Pressure Sensor", Sensors, Vol. 18, No. 10, pp. 3279-3293, 2018.

[5] S. I. Siddiqui, and H. Dong, "Time diversity passive time reversal for underwater acoustic communications", IEEE Access, Vol. 7, pp. 24258-24266, 2019.

[6] T. Lo, "Maximum Ratio transmission", IEEE Transaction on Communication, Vol. 47, No. 10, pp. 1458-1461, 1999.

[7] T. Dubois, M. Crussiere, and M. Helard, "On The Use of Time Reversal for Digital Communications with Non-impulsive Waveforms", In: Proc. of 2010 4th International Conference on Signal Processing and Communication Systems, pp. 1-6, 2010.

[8] T. Dubois, M. Helard, M. Crussiere, and C. Germond, "Performance of time-reversal Precoding technique for MISO-OFDM Systems", EURASIP Journal on Wireless Communications and Networking, Vol. 260, No.1, pp. 1-16, 2013.

[9] D. Love and R. Heath, "Equal Gain Transmission in Multiple-Input MultipleOutput Wireless Systems", IEEE Transaction on Communication, Vol. 51, No. 7, pp. 11021110, 2003.

[10] C. Oestges, A. Kim, G. Papanicolaou, and A. Paulraj, "Characterization of Space-Time Focusing in Time-Reversed Random Fields", IEEE Transaction on Antennas and Propagation, Vol. 53, No. 1, pp. 283-293, 2005.

[11] D. Abbasi-Moghadam and V. T. Vakili, "A SIMO One-Bit Time Reversal for UWB Communication Systems", EURASIP Journal on Wireless Communications and Networking, Vol. 113, No. 1, pp. 1-10, 2012.

[12] B. Wang, Y. Wu, F. Han, Y.H. Yang, and K. Liu, "Green Wireless Communications: A Time-Reversal Paradigm", IEEE Journal on Selected Areas in Communications, Vol. 29, No. 8, pp. 1698-1710, 2011.

[13] S.Q. Xiao, J. Chen, B.Z. Wang, and X.F Liu, "A numerical Study on Time Reversal Electromagnetic Wave for Indoor Ultrawideband Signal Transmission", Progress in Electromagnetics Research, Vol.77, pp.329342, 2007.

[14] T. Dubois, M. Helard, M. Crussiere, and I. Maaz, "Time Reversal Applied to Large MISOOFDM Systems", In: Proc. of 2013 IEEE 24th International Symposium on Personal Indoor and Mobile Radio Communications, London, United Kingdom, pp. 896-901, 2013.

[15] M. Maaz, M. Helard, P. Mary, and M. Liu "Performance Analysis of Time-Reversal Based Precoding Schemes in MISO-OFDM", In: Proc. of 2015 IEEE 81st Vehicular Technology Conference, pp.1-6, 2015. 
[16] Y. Widiarti, Suwadi, Wirawan, and T. Suryani, "A Geometry-Based Underwater Acoustic Channel Model for Time Reversal Acoustic Communication", In: Proc. of the IEEE International Seminar on Intelligent Technology and Its Applications, pp. 345-350, 2018.

[17] Y. Widiarti, Wirawan, and Suwadi, "Image Transmission with Joint Time Reversal and OFDM in Underwater Acoustic Environment", Journal of Physics: Conference Series, Vol. 1179, No.1, pp.1-6, 2018.

[18] D. Abbasi-Moghadam and V.T. Vakili, "A Single Input-Multiple Output Time Reversal UWB Communication System", Wireless Personal Communications, Vol. 66, No. 2, pp. 367-383, 2012.
[19] T. Dubois, M. Helard, and M. Crussiere, "Time Reversal in A MISO OFDM System: Guard Interval Design, Dimensioning, and Synchronisation Aspects", In: Proc. of Wireless World Research Forum, pp. 1-6, 2012.

[20] T. B. Santoso, E. Widjiati, Wirawan, and G. Hendrantoro, "Parameter Measurement of Acoustic Propagation in The Shallow Water Environment", In: Proc. of International Conference on Information Technology and Electrical Engineering, pp. 231-234, 2012.

[21] D. Mitić, A. Lebl, B. Trenkić, and Ž. Markov, "An Overview and Analysis of BER for Three Diversity Techniques in Wireless Communication Systems", Yugoslav Journal of Operations Research, Vol. 25, No. 2, pp. 251269, 2015. 